\title{
Multiframe Interferometry Diagnostic for Time and Space Resolved Free Electron Density Determination in Laser Heated Plasma
}

\author{
A. Blažević, ${ }^{* 1}$, G. Schaumann ${ }^{2}$, A. Frank ${ }^{2}$, T. Heßling ${ }^{1}$, A. Pelka ${ }^{2}$, A. Schökel ${ }^{2}$, D. Schumacher ${ }^{2}$, \\ D.H.H. Hoffmann ${ }^{2}$ and M. Roth ${ }^{2}$
}

${ }^{I}$ GSI Helmholtzzentrum für Schwerionenforschung GmbH, Darmstadt, Germany

${ }^{2}$ Technische Universität Darmstadt, Karolinenplatz 5, D-64289 Darmstadt, Germany

\begin{abstract}
Whereas the energy loss of ions penetrating cold matter is understood and several theories, codes and tables exist, the interaction with plasma is scarcely investigated and only a few experimental data exist. Therefore the interaction of heavy ions penetrating hot and dense plasma is explored at the GSI Helmholtzzentrum für Schwerionenforschung using powerful lasers to create a plasma and ions from the UNILAC accelerator to probe the target. For the interpretation of the experimental data it is crucial to know the plasma parameters like density and temperature as a function of time and space. Therefore a multiframe laser interferometry has been developed to fulfil the requirements. The set up of the interferometry is presented as well as some results of the free electron density distribution of expanding carbon and aluminium plasma at different times.
\end{abstract}

Keywords: Multiframe wollaston interferometry, laser, plasma, diagnostic, free-electron density.

\section{INTRODUCTION}

Since the discovery of fission fragments in the beginning of the 20th century the interaction of heavy ions with solid or gaseous matter has been investigated intensively. The pioneering theoretical work by Bethe [1,2] Bloch [3] and Bohr [4] founded a theoretical base for the understanding of ion-matter interaction and the stopping power of matter, while experiments resulted in a number of semi- or empirical formulas, tables or codes for the stopping power [5-9], or the mean charge of the projectile and its charge state distribution [10-13]. Despite the vast amount of theoretical and experimental work, a proper general microscopic description of the physical processes has started only during the last decade [14-17] and needs more improvement.

Even worse is the detailed knowledge of the interaction of ions with plasmas, a field with increasing interest especially for astrophysics, where plasma is the usual state of matter. Fusion physics is another field that features great interest for beam plasma interaction since accelerators are a candidate for a primary driver in inertial confinement fusion [18], where a small pellet, filled with a deuterium-tritium mixture, is heated by X-rays in a hohlraum, or as final igniter of an already compressed fuel capsule [19].

Early stopping power experiments for protons and heavy ions in ionized matter have used discharge plasma and Zpinch plasma [20-24]. The experiments using gas discharge plasma as stopping medium reached densities up to $10^{17}$ free electrons $/ \mathrm{cm}^{3}$ and plasma temperatures of a few eV for hydrogen plasma [25-27]. The results show a clearly enhanced stopping power of the projectiles in the plasma

*Address correspondence to this author at the GSI-Helmholtzzentrum für Schwerionenforschung GmbH, Darmstadt, Germany; Tel: +49 6159 712294; Fax: +49 6159 712992; E-mail: A.Blazevic@gsi.de compared to cold matter. In contrast to cold matter the projectiles do not interact with neutral atoms but with target ions and additionally with a free electron gas. Stopping power models applicable for this experimental situation can be based on theories given by Bethe, Bohr and Bloch. The situation for partially ionized plasmas can be described in the frame of the standard stopping model $[28,29]$, based on the Bethe formalism as suggested e.g. by T. Peter [30]:

$$
-\frac{d E_{p}}{d x}=\frac{16 \pi a_{0}^{2} I_{H}^{2} Z_{p}^{2} e f f}{m e v_{p}^{2}}\left[\sum_{Z=0}^{Z_{t}}\left(Z_{t}-Z\right) n_{Z} \ln \left(\frac{2 m_{e} v_{p}^{2}}{\bar{I}_{Z}}\right)+n_{e} \ln \left(\frac{2 m_{e} v_{p}^{2}}{\hbar \omega_{p l}}\right)\right]
$$

with $a_{0}$ : the Bohr radius, $I_{\mathrm{H}}$ : the mean ionization potential of hydrogen, $Z_{\mathrm{p}, \text { eff: }}$ effective projectile charge, $m_{\mathrm{e}} v^{2}$ : electron mass times projectile velocity, $Z_{\mathrm{t}}$ : target atomic number, $n_{Z}$, $n_{\mathrm{e}}$ : density of plasma ions of charge $Z$ and free electrons, respectively, $\bar{I}_{Z}$ : the mean excitation energy of the target ion with charge $Z$, and $\hbar \omega_{\mathrm{pl}}$ : energy of the target plasmon. The Coulomb logarithm is split up into two contributions. The first term describes the energy transfer to the remaining bound electrons in the plasma. As in a plasma a charge state distribution is created it is necessary to sum over all charge states $Z$ and take into account the varying mean ionization potential of the different charge states. In the second term the contribution of free electrons is described. Mainly two effects contribute to the stopping power enhancement of ions in fully ionized hydrogen plasma:

(a) A more efficient energy transfer to the free electron gas.

For free electron densities below $10^{21} \mathrm{~cm}^{-3}$ the plasmon energy $\hbar \omega_{\mathrm{pl}}$ is less than $1 \mathrm{eV}$, thus much smaller than the average of the excitation or ionization energy of bound electrons in neural atoms. This leads to an increase of the stopping power. 
(b) An increase of the projectile charge state.

Since for a free electron it is nearly impossible to fulfil energy conservation and momentum conservation at the same time in a capture process, the dynamic equilibrium of capture and ionization processes is shifted towards a higher projectile charge states, which in turn leads to an increase of the mean and herewith the effective charge $Z_{\mathrm{p}, \text { eff }}$.

The stopping power of ionized matter depends strongly on $Z_{\mathrm{p} \text {,eff. }}$ There is no simple relation between $Z_{\mathrm{p} \text {,eff }}$ and the charge state of the projectile. The experimental results and the arguments given above suggest however, that the charge state of an ion traversing fully ionized hydrogen plasma is higher than the charge state of the projectile under the same conditions of density in cold hydrogen gas. In different laboratories experiments were carried out to measure and calculate the charge state of ions passing through ionized matter [31-34].

\section{EXPERIMENTAL SET UP FOR ENERGY LOSS MEASUREMENT OF IONS PENETRATING PLASMA}

The GSI plasma physics group has dedicated their efforts to extend the experimental data base for the stopping power of ions in plasmas to higher densities and higher temperatures. At the experimental area Z6, a branch of the UNILAC (Universal linear accelerator), an experimental setup has been built up for the investigation of the interaction of ions with laser generated plasma [35]. Therefore a thin foil, usually a carbon foil with a thickness of $100 \mu \mathrm{g} / \mathrm{cm}^{2}$, is irradiated by the nhelix [36] or the Phelix [37] laser (amplified Nd: YAG laser with $\lambda=1064 / 1053 \mathrm{~nm}$, energy up to $100 \mathrm{~J}$ in 5-15 ns (FWHM)) transforming the foil into a dense and hot plasma with densities up to a few percent of solid state density and temperatures over $200 \mathrm{eV}$ in the hottest area (see Fig. 1). This geometry, with a large laser focus spot size ( $1 \mathrm{~mm}$ diameter generated by a random phase plate), twice as large as the ion beam, has been chosen to create a plasma, that is transversally homogeneous for the ion beam and that is expanding at least for some ns almost one-dimensionally in its centre. Hence, in contrast to experiments described in e.g. [22], where the plasma expands orthogonally to the probing ion beam there are density and temperature gradients only along the ion beam axes, which have to be determined experimentally.

At the same time the plasma is generated, an ion pulse with a length of a few tens $\mu$ s, built up of micro bunches with a length of $3 \mathrm{~ns}$ FWHM and a frequency of $108 \mathrm{MHz}$, is probing the plasma parallel to its expension direction. This means the expanding plasma is being investigated every 9.2 ns. The delay between the laser and the ion bunch can be shifted with an accuracy of $1 \mathrm{~ns}$. The ions first penetrate a colder part of the ionized foil, and then enter dense and hot plasma, which is expanding and cooling. Finally, after several hundreds of ns, all matter along the interaction path has expanded so far that no energy loss is measurable and the ions fly through vacuum with their initial energy. The energy loss as a function of time is determined by a time of flight measurement. Additionally a dipole magnet behind the target chamber can be used to measure the charge state

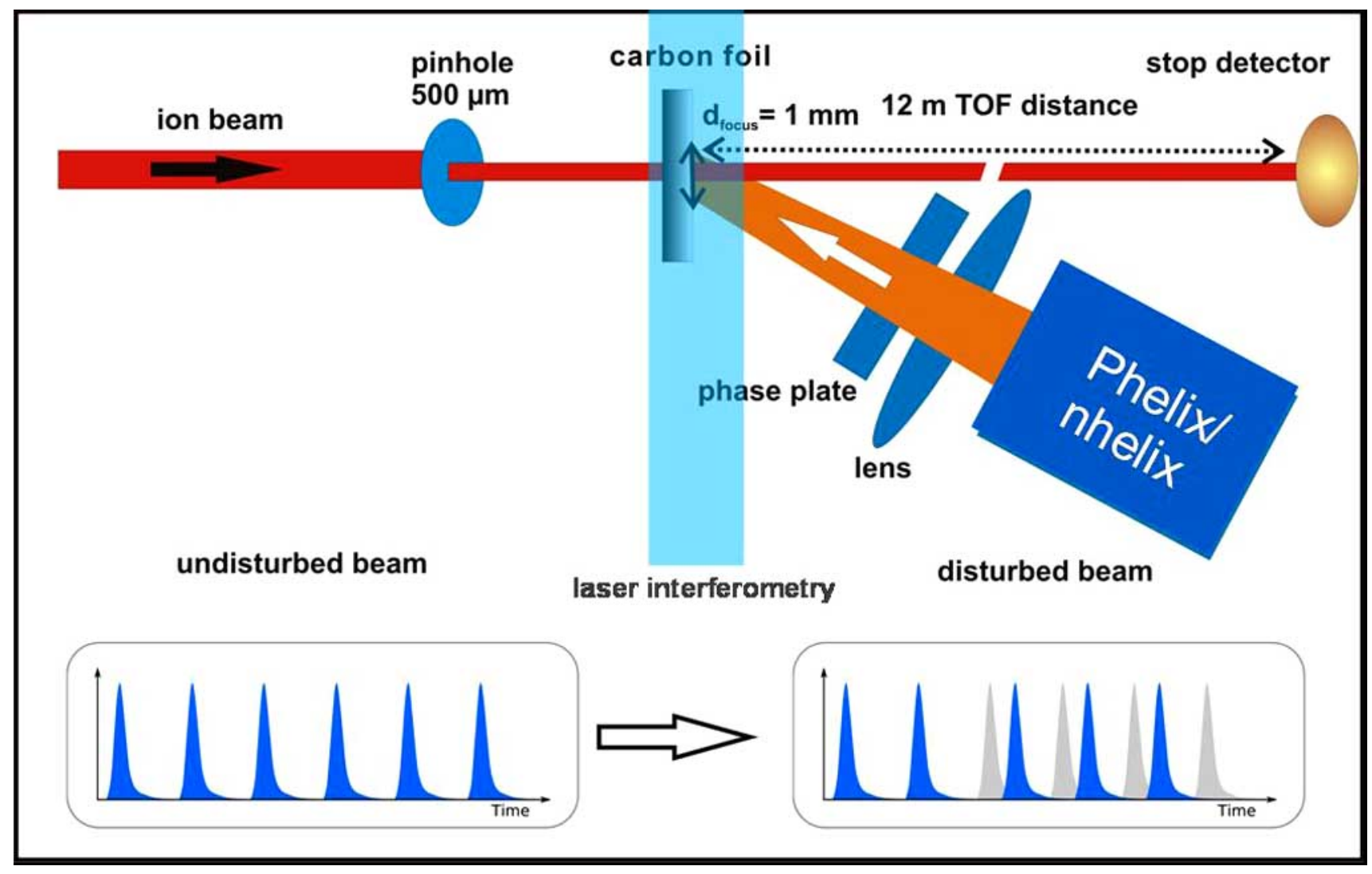

Fig. (1). Experimental setup for the interaction experiments of ions with laser driven plasma. 
distribution of each single bunch penetrating the plasma. As a result we obtain a set of energy loss data and charge state distributions of ions probing expanding plasma. As the target conditions with respect to density and temperature during the interaction time are important, a set of plasma diagnostic tools is used e.g. laser interferometry for a space resolved measurement of the free electron density $n_{\mathrm{e}}$, time resolved Xray spectroscopy for the temperature determination, a visible streak camera measuring the expansion velocity, pinhole cameras, etc. Nevertheless, the very dense and cold part of the plasma, being too dense for laser penetration and too cold and opaque for radiation spectroscopy, is not accessible to most of these diagnostics. Therefore results of the plasma diagnostic serve to benchmark hydrodynamic simulation of the laser-matter interaction and plasma expansion. From these simulations the necessary density and temperature profiles along the ion path can be extracted for the theoretical calculation of the energy loss and charge state distributions.

\section{MULTIFRAME INTERFEROMETRY}

The problem described above requires plasma diagnostics sophisticated enough to determine key plasma parameters such as temperature and free electron density with both space and time resolution during the interaction time. Hence an interferometer was developed and constructed to determine the electron density with spatial resolution and simultaneously at different times in a single laser shot, leading to a time resolution of the expanding free electron density.

This diagnostic provides images of the interference pattern with a time difference of $1.5 \mathrm{~ns}$ and allows determination of the free electron density up to a maximum density of $3 \times 10^{20} \mathrm{~cm}^{-3}$. Fig. (2) shows the experimental setup of the multiframe interferometry. Before the interaction of the probe beam with the plasma, a pulse of the $\mathrm{Nd}$ : YAG laser with a pulse length of $500 \mathrm{ps}$ is frequency tripled, resulting in a wavelength of $355 \mathrm{~nm}$. This wavelength corresponds to a critical electron density $\left(\mathrm{n}_{\mathrm{ec}}\left[\mathrm{cm}^{-3}\right] \approx 1 \cdot 10^{21} / \lambda^{2}[\mu \mathrm{m}]\right)$ of $8 \cdot 10^{21} \mathrm{~cm}^{-3}$ up to which the probe beam theoretically can permeate the plasma. In practice, the actually measurable maximum electron density is lower.

The time resolution of every single image is given by the $0.5 \mathrm{~ns}$ (FWHM) pulse duration of the diagnostic laser, while the time in between two frames corresponds to the time of circulation in the optical ring (see Fig. 2). As the pulse is circulating in the ring a half wave plate gradually turns the polarization direction at each turn and part of the pulse energy is transmitted at the second polarizer. The inset image in Fig. (2) shows the pulse train generated in the optical ring. In order to be able to separate these pulses spatially, the ring is adjusted so that the subsequent pulses leave the ring under a small angle with respect to each other. At the plasma position the different beams sufficiently superimpose, while in the far field, at the focal position of the first imaging lens, it is possible to separate the subsequent pulses with micro mirrors. A separate Wollaston interferometer for each pulse measures the optical path difference induced by the interaction with the plasma, which allow for the calculation of the free electron density.

\section{EXPERIMENTAL RESULTS}

Fig. (3) illustrates spatial electron density distributions

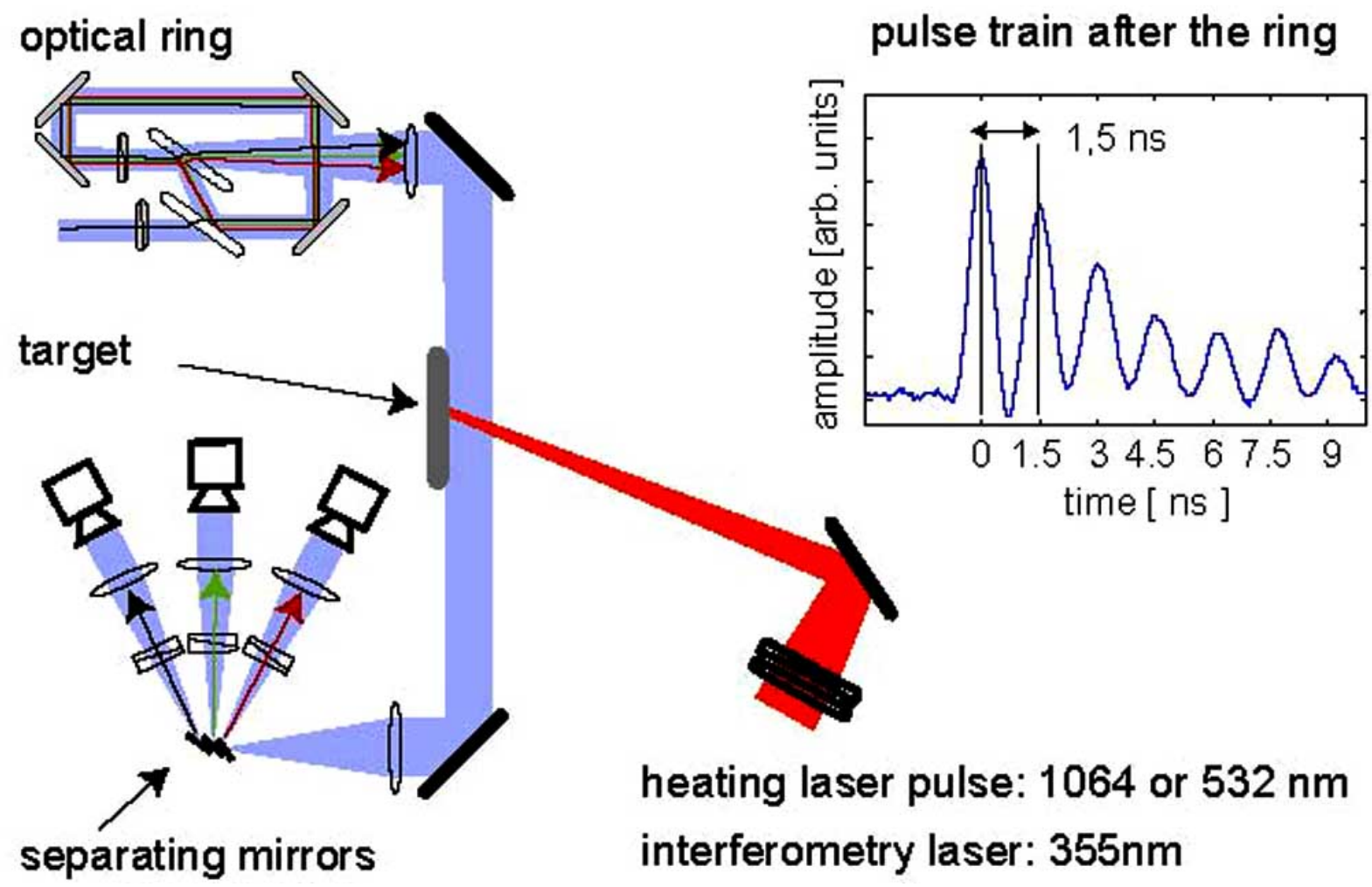

Fig. (2). Experimental setup of the multiframe interferometry. 


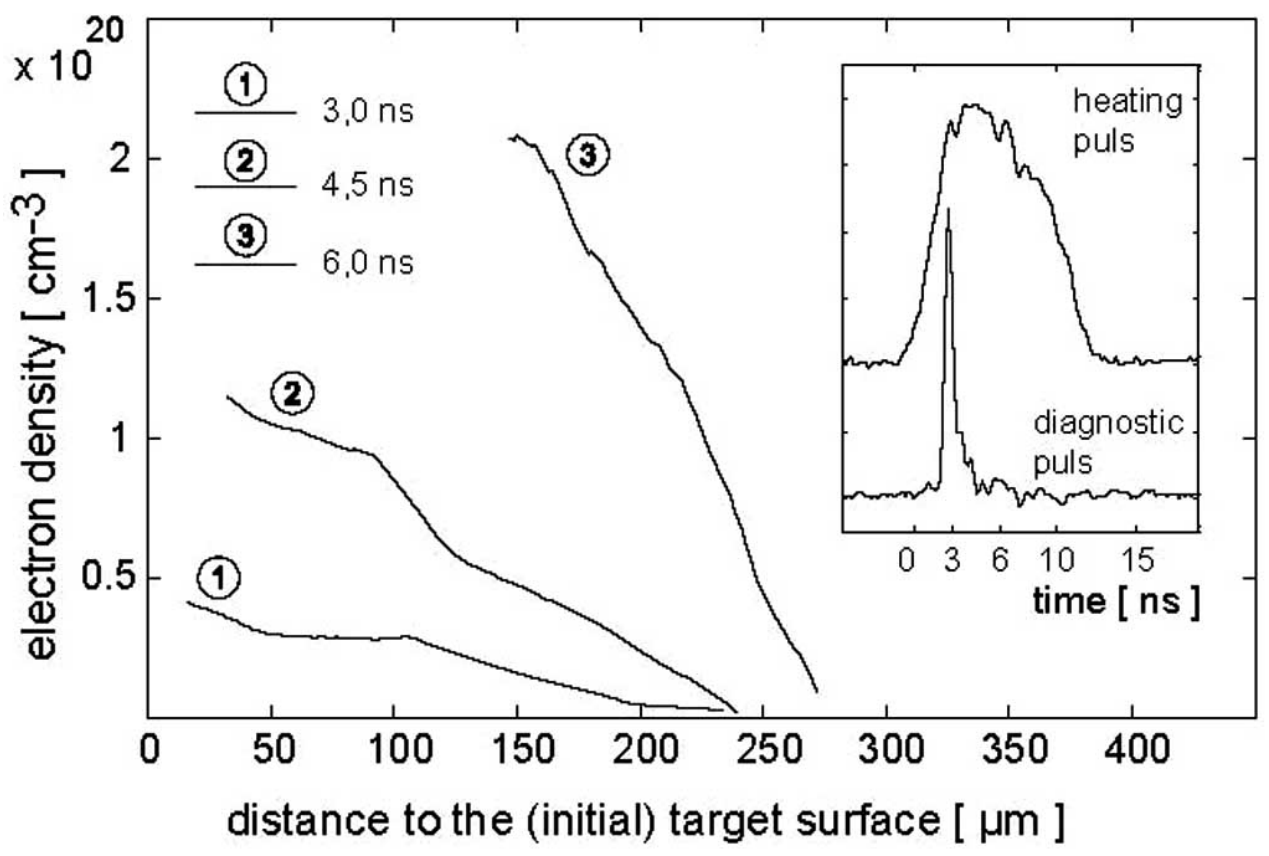

Fig. (3). Spatial electron density distribution for three frames.

perpendicular to the target surface at the centre of the Gaussian focal spot of the heating laser pulse. The three distributions correspond to the first three pulses and show the time evolution of the free electron density measured at the same experiment, within a single shot of the plasma generating laser, with the following parameters:

- $\quad$ laser energy: $57 \mathrm{~J}$
- $\quad$ wave length: $1064 \mathrm{~nm}$

- $\quad$ pulse length: $10 \mathrm{~ns}$ (see inset in Fig. (3))

- $\quad$ focus diameter: $1 \mathrm{~mm}$

- $\quad$ target: $100 \mu \mathrm{g} / \mathrm{cm}^{2}$ carbon foil

For the experiment depicted in Figs. $(\mathbf{4}, \mathbf{5})$ an Al foil with a thickness of $205 \mu \mathrm{g} / \mathrm{cm}^{2}$ was irradiated with the Phelix laser ( $\lambda=1053 \mathrm{~nm}$, energy: $119 \mathrm{~J}$, pulse lengths: $13 \mathrm{~ns}$

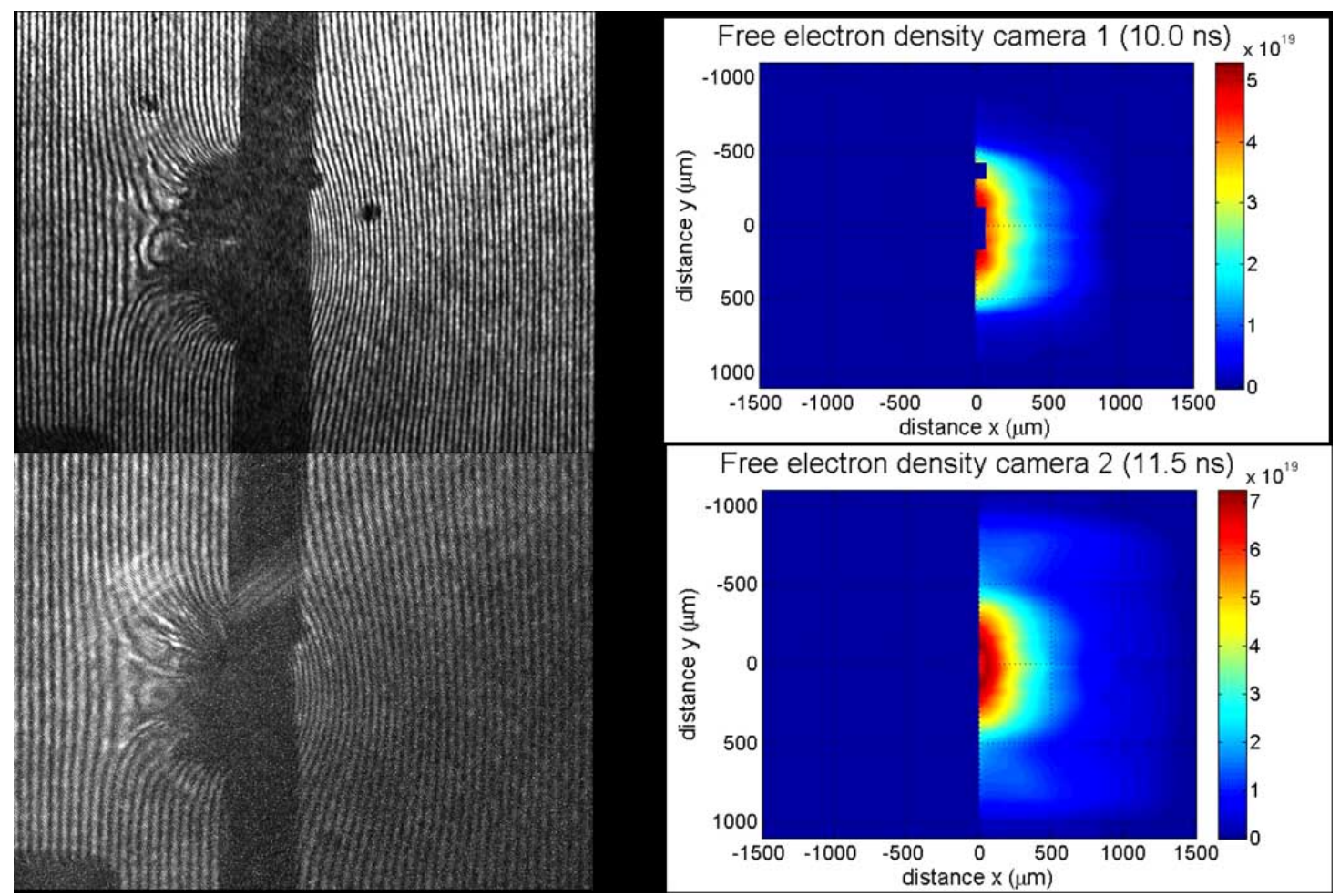

Fig. (4). Interferometry pattern and deconvoluted free electron density of an expanding Al plasma heated with the Phelix laser. 


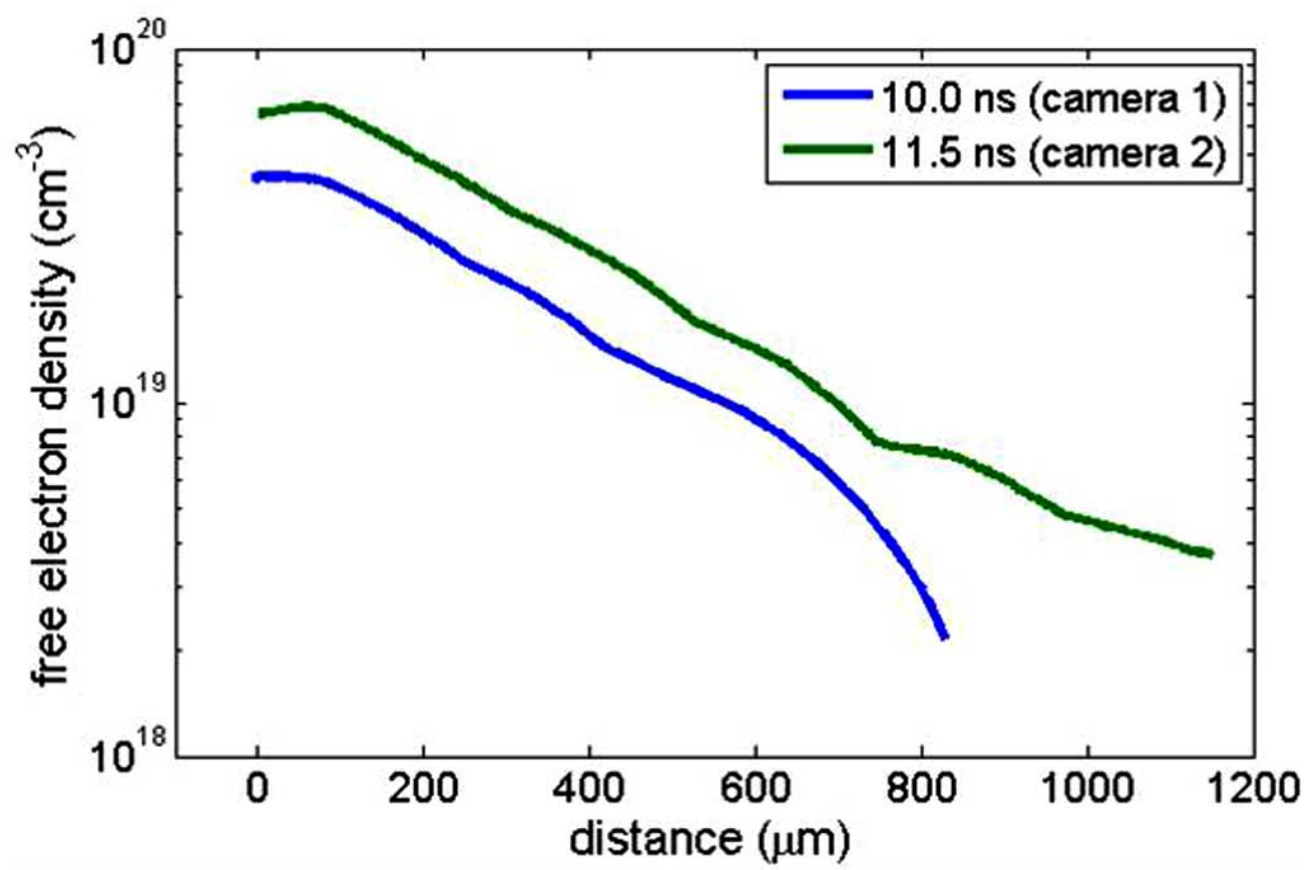

Fig. (5). Free electron density distribution of the expanding Al plasma along the $y=0$ axes at two different times.

FWHM, focal spot diameter: $1 \mathrm{~mm}$ ). Fig. (4) shows on the left side the fringe shifts in the interferometry pattern due to a refraction index dependence of the free electron density. The laser irradiates the target from the right side. The vertical dark shadow emerges from the target holder. The foil was mounted on the right side. After $10 \mathrm{~ns}$ the ablation pressure has pushed the foil back for a few hundreds $\mu \mathrm{m}$, so it appears on the left side. This means, that $\mathrm{x}=0$ is the initial position of the target foil at the time $t=0 \mathrm{~ns}$, it is not the foil surface at times later than $10 \mathrm{~ns}$. The pictures on the right side show the free electron distribution after deconvolution of the phase shifts extracted from the pictures on the left. Taking line outs at $\mathrm{y}=0$, which is the middle of the Gaussian laser intensity distribution, one gets curves like shown in Fig. (5). This kind of data or even the complete 2-D results as shown in Fig. (4), can serve now as a hard benchmark for hydrodynamic simulations.

\section{REFERENCES}

[1] Bethe H. Zur Theorie des Durchgangs schneller Korpuskularstrahlen durch Materie. Ann Phys 1930; 5: 324-400.

[2] Bethe HA. Bremsformel für elektronen relativistischer geschwindigkeit. Z Phys 1932; 76: 293.

[3] Bloch N. The slow down of rapidly moving particles in their passing through solid matter. Ann Phys (Leipzig) 1933; 16: 285.

[4] Bohr N. The penetration of atomic particles through matter. Mat Fys Medd Dan Vidensk Selsk 1948; $18: 8$.

[5] Lindhard J, Scharff M. Energy dissipation by ions in the $\mathrm{keV}$ region. Phys Rev 1961; 124: 128.

[6] Northcliff LC, Schilling RF. Range and stopping-power tables for heavy ions. Nucl Data Tables A 1970; 7: 233-463.

[7] Ziegler JF, Biersack JP, Littmark U. The stopping and ranges of ions in solids. New York: Pergamon Press 1985.

[8] Hubert F, Bimbot R, Gauvin H. Range and stopping power tables for $2.5-500 \mathrm{MeV7nucleon}$ heavy ions in solids. Atomic Data Nucl Data Tables 1990; 46: 1-213.

[9] Stopping of ions heavier than helium: ICRU report no. 73. J ICRU 2005; 5: 1-253.

[10] Shima K, Ishihara T, Mikumo T. Empirical formula for the average equilibrium charge-state of heavy ions behind various foils. Nucl Instr Methods 1982; 200: 605.
[11] Shima K, Kuno N, Yamanouchi M, Tawara H. Equilibrium charge fractions of ions of $Z=4-92$ emerging from a carbon foil. Atomic Data Nucl Data Tables 1992; 51(2): 173-241.

[12] Rozet JP, Stephan C, Vernhet D. ETACHA: a program for calculating charge states at GANIL energies. Nucl Instr Methods Phys Res B 1996; 107: 67.

[13] Schiwietz G, Grande PL. Improved charge-state formulas. Nucl Instr Methods Phys Res B 2001; 125: 175-7.

[14] Schiwietz G, Grande PL. An electronic stopping-power program CasP. Available from: URL: http://www.hmi.de/people/schiwietz/ casp.html

[15] Maynard G, Chabot M, Gardes D. Density effects and charge dependent stopping theories for heavy ions in the intermediate velocity regime. Nucl Instr Methods Phys Res B 2000; 139: 164-5.

[16] Sigmund P, Schinner A. Binary theory of electronic stopping. Nucl Instr Methods Phys Res B 2002; 195: 64.

[17] Blazevic A, Bohlen G, Oertzen WV. Stopping power of swift neon ions in dependence on the charge state in the non-equilibrium regime. Nucl Instr Methods B 2002; 190: 64.

[18] Badger B, Arendt F, Beckert K, et al. A Conceptual Heavy Ion Beam Driven Fusion Reactor Study. UWFDM-450 and KfK 3202, Madison, Wisconsin: University of Wisconsin 1981.

[19] Roth M, Cowan TE, Key MH, et al. Fast ignition by intense laseraccelerated proton beams. Phys Rev Lett 2001; 86(3): 436-9.

[20] Ogawa $M$, Oguri $Y$, Neuner $U$, et al. Laser heated $\mathrm{dE} / \mathrm{dx}$ experiments in Japan. Nucl Instr Methods Phys Res A 2001; 464(13): 72 .

[21] Belyaev G, Basko M, Cherkasov A, et al. Measurement of the Coulomb energy loss by fast protons in a plasma target. Phys Rev E 1996; 53(3): 2701.

[22] Couillaud C, Deicas R, Nardin P, Beuve MA, Guihaumé JM, Renaud M. Ionization and stopping of heavy ions in dense laserablated plasma. Phys. Rev E 1994; 49(2): 1546.

[23] Gardes D, Bimbot R, Dumail M, et al. Experimental investigation of beam-plasma interactions enhanced stopping power - Plasma lens effect. Radiat Effects Defects Solids 1989; 110(1-2): 49.

[24] Hoffmann DHH, Weyrich $\mathrm{K}$, Wahl $\mathrm{H}$, et al. Experimental observation of enhanced stopping of heavy-ions in a hydrogen plasma. Z Phys A 1988; 330(3): 339.

[25] Hoffmann DHH, Weyrich K, Wahl H, Gardés D, Bimbot R, Fleurier C. Energy loss of heavy ions in a plasma target. Phys Rev A 1990; 42: 2313.

[26] Weyrich K, Hoffmann DHH, Jacoby J, et al. Energy-loss of heavyions in a hydrogen discharge: Energy-loss of heavy-ions in a 
hydrogen discharge plasma. Nucl Instr Methods Phys Res A 1989; 278(1): 52 .

[27] Jacoby J, Hoffmann DHH, Laux W, et al. Stopping of heavy ions in a hydrogen plasma. Phys Rev Lett 1995; 74: 1550.

[28] Deutsch C. Maynard G, Bimbot R, et al. Ion beam-plasma interaction: A standard model approach. Nucl Instr Meth A 1989; 278(1): 38 .

[29] Deutsch C. Inertial confinement fusion driven by intense ion beams. Ann Phys Fr 1986; 11(1): 1.

[30] Peter T, Meyer.ter-Vehn J. Energy loss of heavy ions in dense plasma. Phys Rev A 1991; 43(4): 1998

[31] Nardi E, Fisher DV, Roth M, Blazevic A, Hoffmann DHH. Charge state of $\mathrm{Zn}$ projectile ions in partially ionized plasma: Simulations. Laser Particle Beams 2006; 24(1): 131.

[32] Kojima M, Mitomo M, Sasaki T, Hasegawa J, Ogawa M. Chargestate distribution and energy loss of 3.2-MeV oxygen ions in laser plasma produced from solid hydrogen. Laser Particle Beams 2002; 20(3): 475.

[33] Golubev A, Turtikov V, Fertman A, et al. Experimental investigation of the effective charge state of ions in beam-plasma interaction. Nucl Instr and Methods Phys Res A 2001; 464(1-3): 247.

[34] Dietrich KG, Hoffmann DHH, Boggasch E, et al. Charge state of fast heavy-ions in a hydrogen plasma. Phys Rev Lett 1992; 69(25): 3623.

[35] Frank A, Blažević A, Grande PL, et al. Energy loss of argon in a laser-generated carbon plasma. Phys Rev E 2010; 81: 026401.

[36] Schaumann G, Schollmeier MS, Rodriguez-Prieto G, et al. High energy heavy ion jets emerging from laser plasma generated by long pulse laser beams from the NHELIX laser system at GSI. Laser Part Beams 2005; 23: 503 .

[37] Witte K, Bagnoud V, Blazevic A, et al. Performance of PHELIX in 2008. GSI Scientific Report 2008; p. 311.

(C) Blažević et al.; Licensee Bentham Open.

This is an open access article licensed under the terms of the Creative Commons Attribution Non-Commercial License (http://creativecommons.org/licenses/ by$\mathrm{nc} / 3.0 /$ ) which permits unrestricted, non-commercial use, distribution and reproduction in any medium, provided the work is properly cited. 